\title{
Puffle-Pod Marine Evacuation System (POMES)
}

\author{
Eda TUMER \\ De Montfort University \\ Leicester, UK \\ ed.tumer@gmail.com
}

\author{
Stefan KUHN \\ De Montfort University \\ Leicester, UK \\ stefan.kuhn@dmu.ac.uk
}

\begin{abstract}
Evacuation systems have always played a crucial part when designing a transport system. The cornerstone of these systems is to get people to safety in the quickest and safest way possible. When it comes to marine systems, the requirements greatly differ in comparison to those on land and in air. On a day with highly inclement and fierce weather, in the middle of the ocean, getting the crew to safety through a chute or a slide would expose the crew to ferocious danger. Thence, the proposed Puffle-Pod Evacuation System introduces a more protected and secure evacuation without putting the lives of the crew at a high risk.
\end{abstract}

Keywords-marine, evacuation system, puffle pod

\section{INTRODUCTION}

Within the last few decades there have been vast changes in evacuation systems and styles of marine vehicles from buoys and (inflatable) boats to life-rafts [1][3][5] and hyperbaric lifeboats [6].

The first Marine Evacuation System (MES) invented in 1979 by RFD was a milestone for marine safety [2][8]. Their current generation of MES allows a highly rapid and safe evacuation of 860 passengers in less than 30 minutes [9]. It is a great success of pace considering the rate of people leaving the ship per minute. The MESs currently in use are -as explained in further detail in the Related Work section- very vulnerable to natural misfortunes.

The main purpose of this paper is to propose a safer and more secure evacuation. The proposed evacuation system, called POMES, introduces a new style of evacuation by presenting pods which encapsulate and isolate the personnel inside, in order to provide as much protection as possible.

The paper is organised as follows. Section II briefly introduces the concept of the existing systems on market. The novel proposal of the paper is described in Section III respectively. Section IV concludes this paper by contrasting briefly the existing systems and POMES.

\section{RELATED WORK}

The probability of survival in an emergency needs to be very high for a naval evacuation system. There are various companies which provide evacuation systems for marine vehicles. These companies provide off-the-shelf systems but offer numerous choices by making them suitable for common types of marine vehicles. The evacuation systems provided by these organisations mainly consist of inflatable rafts which are also called life-rafts [1][2][3]. Some of these rafts are used by different navies across the world [1]. Ostensibly, all the systems use inflatable rafts for evacuation so far. These systems get the crew off the ship through a slide or a chute, which is inflated by a member of the crew by the pull of a handle as soon as the alarm is activated [1] $[2][3][4][5][7]$. These systems depend entirely on inflating those rafts/boats. These life-rafts are safer in contrast to lifeboats which are deployed more commonly in MESs, since they are made with high resistant fibres [2].

Survitec and other famously known providers of evacuation systems provide more than one system depending on the demands and suitability [1][5][3]. Survitec offer 6 groups of Marine Evacuation Systems depending on the size and speed of the vessel [1]. Wärtsilä, another well-known company for MESs, have similar systems as Survitec with a few different features [3]. Yet, as mentioned earlier, these systems use either chutes or slides with inflatable life-rafts. Using chutes or slides may cause injuries -due to landing and sliding positions [15] which eventually affects the safety of these systems.

\section{PUFFLE-POD}

\section{A. System Introduction}

The puffle-pod marine evacuation not only provides a more secure and safer way to get the crew to evacuate the vessel, it also increases the chance of survival of the personnel afterwards in the sense of physical and psychological wellbeing. Post evacuation, the sustenance is also provided.

The purpose of designing the puffle-pods in a way that they are accessed from inside the vessel is to protect the crew from potentially harmful external happenings. Sustenance and additional support for subsistence, such as provision of a fishing rod, can be provided inside every pod. Another key benefit of using these pods is that the personnel would be kept warm and dry, which avoids likeliness of disease caused by cold and staying wet.

Also, having other people inside the pod could support the individual by not letting them feeling isolated or alone. The personal space provided by the pods greatly decreases anxious feelings caused by overcrowdedness. In contrast to the existing MESs, POMES grants people the personal space they need. The distress caused by the discomfort of having

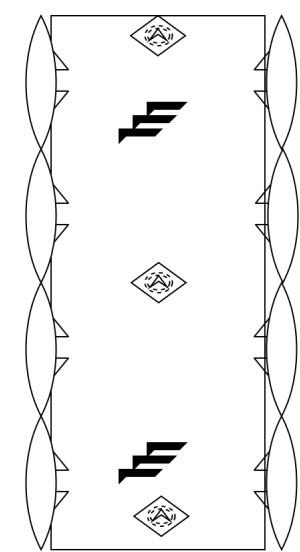

Figure 1 - Deflated to let other people be too close would proportionally increase with time. Thus provision of minimum personal space is given by dividing 45 personnel into 4 pods, in case that half the pods are damaged or not functioning. Hence in the best case scenario, in which the pods have only 5-6 people inside, the crew are not perturbed by the vile feeling of letting other people into their personal space. This is only an exemplary design. This layout can be adjusted for other sizes of ships, taking into account relevant standards for evacuation of marine systems [14]. 


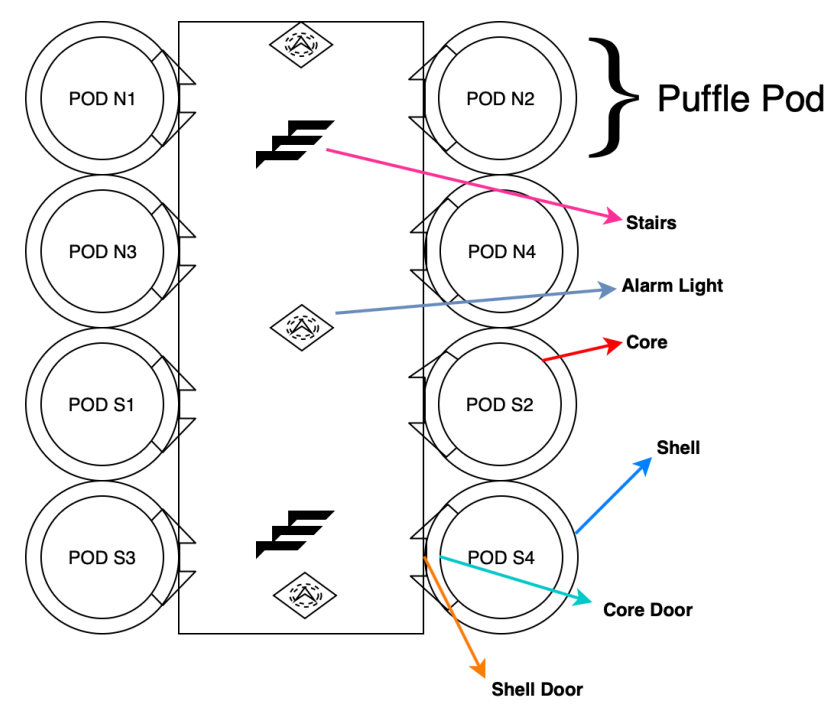

Figure 2 - How pods look like in the vessel

\section{B. Functionality Description}

The proposed POMES is designed for evacuation of crew of navy vessels which have a capacity of 45 personnel. This system prepares the pods when evacuation is taking place, and leads each person to an empty pod.

POMES consists of 8 pods on each side of the vessel. Each pod has a capacity of 12 personnel at maximum. In normal conditions, the pods are deflated in order to make them conducive to save space outside the vessel (Figure 1).

A puffle-pod consists of two ball-like shaped balloons one inside the other. The outer balloon is called the shell and the other one is called the core (Figure 2). The shell shall be made of a tougher and more resistant fibre tissue, in contrast to the core, in order to protect the pod from possible bites or damage from marine creatures. The material of the shell, therefore, shall be made of a lightweight, durable and cutresistant material, such as Kevlar [11][17] or auxetic Kevlar $^{\circledR}$ [18]. The space between the core and the shell at the bottom of the pod is filled with sand, or any other material which does not float and considerably heavy but flexible, in order to prevent disorientation in the sea; and the rest of this space is filled with air. The cover of the core shall be made of a material, such as Nomex [10][17][14], which is flame resistant and has excellent thermal resistance, which would keep the crew warm by protecting them from the cold.

The preparation of pods starts with the activation of the main inflators, which consequently activates the pods' individual inflators. The main inflators are more powerful in comparison to the pods' inflators. The reason of this implementation is to get the air into the pods as quick as possible.

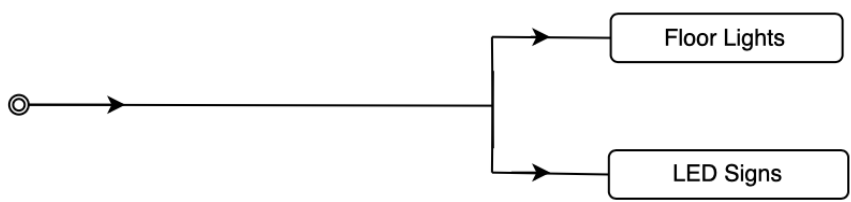

Figure 3 - Pressure Sensor / Light Connexion

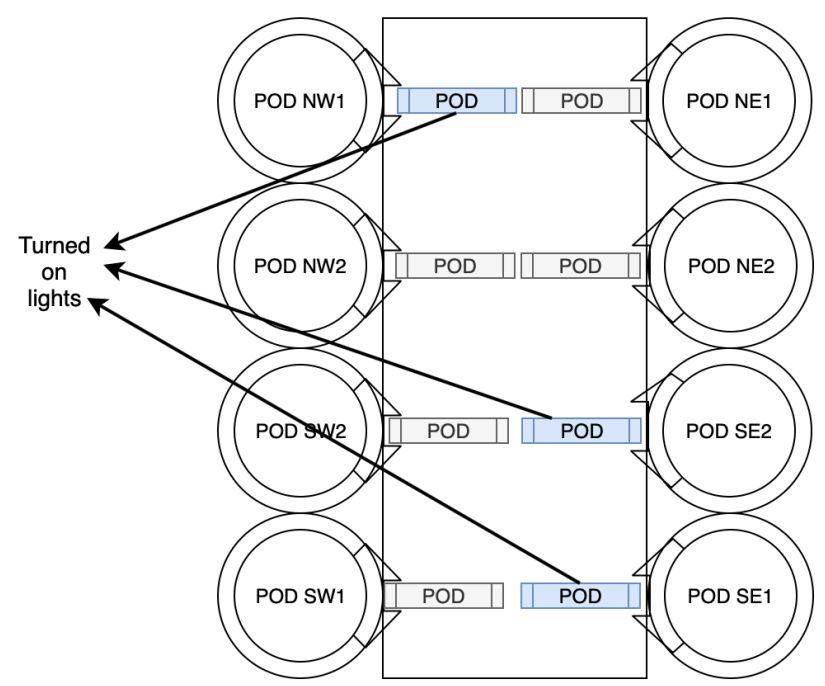

Figure 4 - Setting of the floor and LED lights

In the event of reaching the right amount of air pressure inside a pod, it is signalled to the controller via the pod's pressure sensor that the pod is ready. Consequently, the pod's inflator is stopped and the pod's door is unlocked and opened. The pressure sensor also sends signals to the floor and LED signs of the pod to turn them on (Figure 3).

Each pod has an LED sign in the vessel and floor lighting leading to the pod. These lights get activated whenever a pod is ready and get turned on and off according to the pods' availability by the signal coming from pressure sensors of the pods. The pods' doors work in the same way, they get locked or unlocked and opened in accordance with the preparation of the pods. Thence, the crew are directed straight to the prepared pods by these lights.

The sequence of the whole evacuation event, therefore, shall ideally be as follows:

$i$ - The emergency takes place and the evacuation system is triggered.

ii- The controller receives the signal and sends the signal to activate the main inflators.

iii- All the other inflators get activated.

iv- When a pod reaches a certain amount of pressure, the pressure sensor of the pod then:

(a) sends a signal to the controller

(b) turns on the floor and LED sign lights belonging to the pod

$\boldsymbol{v}$ - Once the controller receives positive signals from the pressure sensors, it unlocks and opens the doors of the ready pods.

vi- The controller turns off the inflators after certain amount of time / when it starts to receive signals from pressure sensors.

vii- When the crew decide to separate themselves from the vessel, they have to close the vessel's and the pod's doors.

viii- The pods get separated from the vessel manually by of the crew inside them.

ix- Separation from the vessel means that the blockage between the battery and the transmitter inside the pod is 


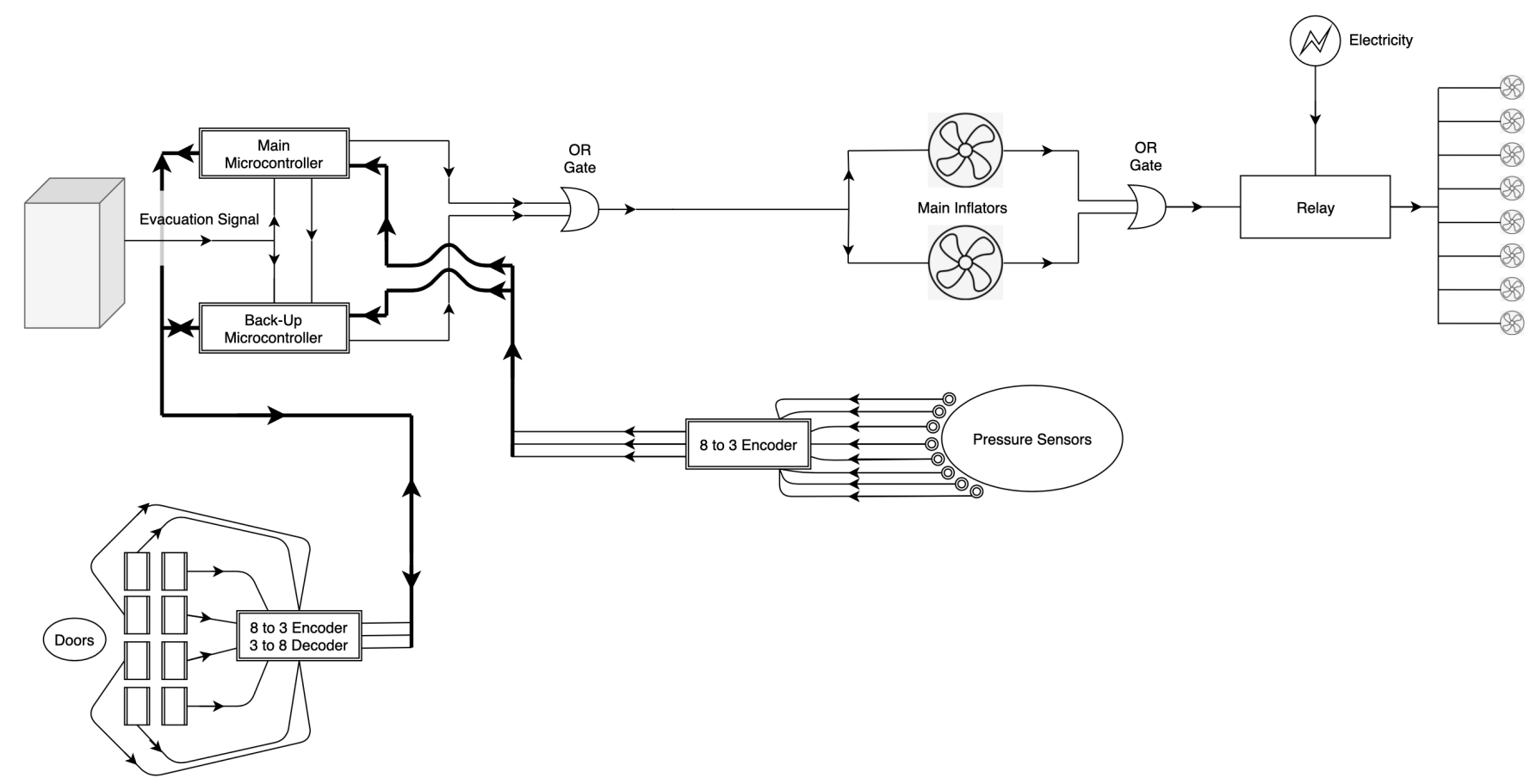

Figure 5 - Overall view of POMES

removed. Hence the transmitter starts to transmit S-O-S signals upon receiving power.

$\boldsymbol{x}$ - When a pod separates itself from the vessel:

(a) The vessels door is locked by the same operation which separates the pod from the vessel

(b) The lights which lead to the separated pod are turned off in order to prevent misleading the personnel that are still inside the vessel.

\section{Technical Description}

The controller is implemented by two microcontrollers. The primary microcontroller is called the main microcontroller. The main microcontroller and the secondary microcontroller, called the back-up controller, are connected to each other. When the back-up microcontroller stops receiving any signal from the main microcontroller, it takes control of the system and continues on with the process. These microcontrollers are to be positioned oppositely, meaning that one has to be at the front and the other at the rear of the vessel. It would let the system continue functioning if for instance a collision occurs where the main microcontroller is positioned.

As soon as the evacuation signal is created, it reaches both the main microcontroller and the back-up microcontroller. The first OR gate on the way to the inflators is put there in order to simplify the system. Thus the rest of the system do not have to be aware of which microcontroller is in charge. The second OR gate's purpose is to activate the pods' inflators if any of the main inflators are functioning. This is to accommodate any failure of either the main inflators.

The reason for the relay following the OR gate is to multiply and to activate the rest of the inflators. Whenever a signal reaches the relay, the relay makes the connexion between electricity and the rest of the system. Upon receiving a current, the rest of the system would be powered on, hence the pods' inflators start working.
After a certain amount of time all the pods are expected to reach the sufficient air pressure. Each pod has a pressure sensor in order to monitor the air pressure. These sensors are connected to the microcontrollers through an encoder. The encoder reduces the number of cables immensely. In the case that the encoder is not used, there has to be 16 cables in total instead of 6 because every sensor has to be connected to both controllers hence we have to double the numbers.

The connexions between the doors and the microcontrollers are done in the same way, using an encoder, for the same purpose mentioned earlier. In addition to this, in order to control the doors, 3 outputs are produced by the microcontroller in charge. Just like the signals encoded into 3 when delivering the status of the doors to the MCU(microcontroller unit), the outputs of the microcontroller are delivered to a 3 to 8 decoder, and wired to the doors accordingly. The doors are controlled by the controller and not by pressure sensors like lights, because they have more than 2 states and these states have to be monitored and handled with scrutiny.

The pseudocode of these microcontrollers are shown as follows (it has to be taken into consideration that all the doors are locked as default).

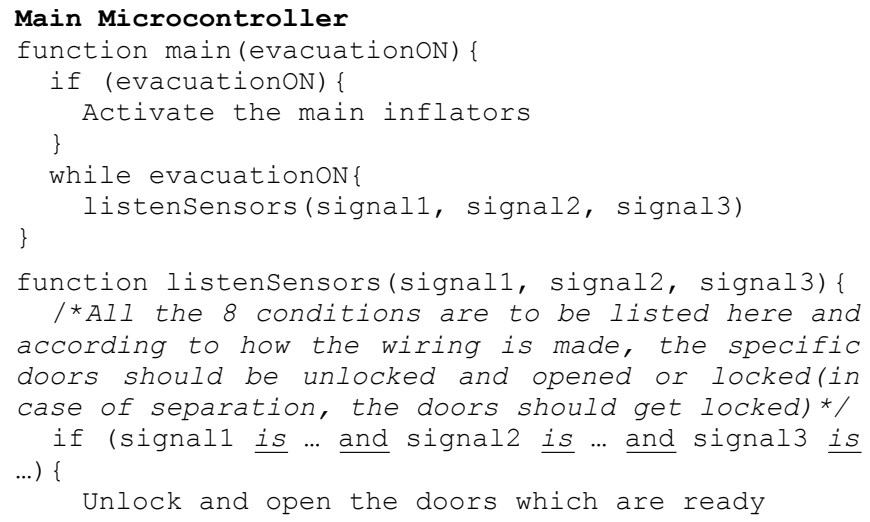




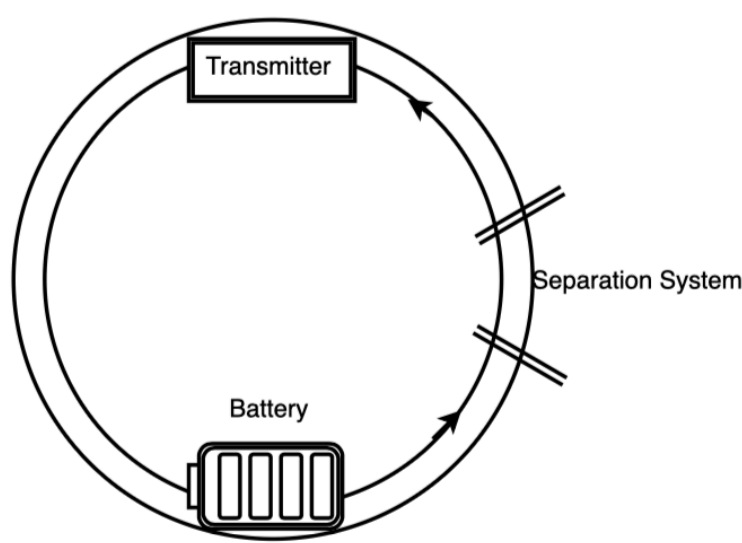

Figure 6 - The system inside the pod

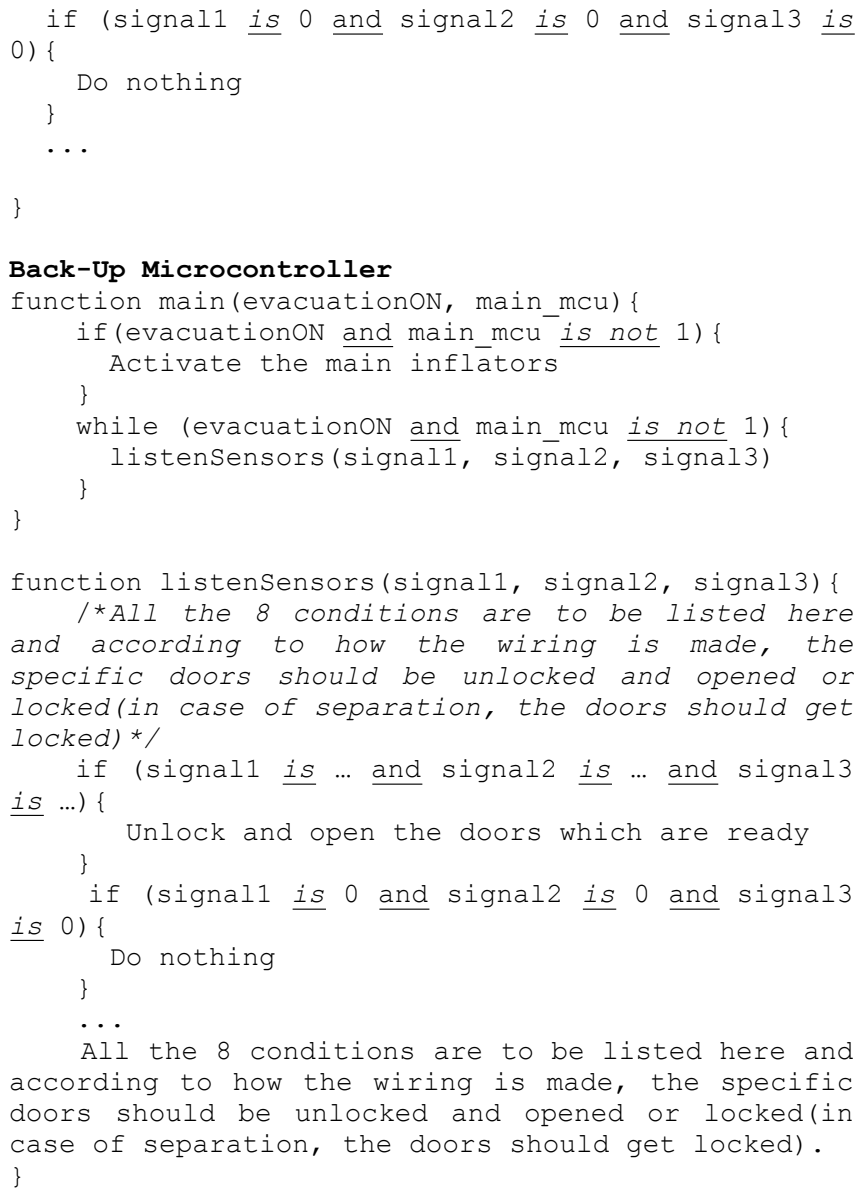

The separation system on the pod's side blocks the energy current which tries to flow from the battery along way to the transmitter(Figure - 6). Upon separation form the vessel, the blockage between the battery and the transmitter no longer exists, thence the transmitter starts receiving power. Consequently, S-O-S signals are transmitted.

\section{Critical Condition Analysis}

In order to establish the safety of the proposed system, the recommended techniques in IEC 61508 for Functional Safety of Electrical/Electronic/Programmable Electronic Safety-related Systems [12] (of which POMES is an example) and ISO/IEC 12207 for Systems and software engineering [13] are used. A major activity is hazard identification, followed by risk assessment, leading to risk analysis and mitigations for the identified relevant hazards.
The system suggested here uses many techniques to control risks, as explained above. Here, some of the steps, which are taken during the design and development process, are defined. A full documentation is out of scope for this overview paper.

\section{a. Hazards}

a1. POMES not activated. (Intolerable)

a2. A pod is not inflated (ALARP)

a3. A door of a pod not unlocked. (ALARP)

a4. A door of a pod not opened. (ALARP)

a5. LED signs or floor lights of a pod not turned on. (ALARP)

a6. A pod does not detach when released. (Intolerable)

a7. Pods' doors opened when should not. (Intolerable)

We assess some risks as intolerable, whereas others fall into the "as low as reasonably practicable" range. This is because the number of pods already provides redundancy, and individual failures can be tolerated to a very low degree.

\section{b. Causes of the Hazards}

a1.1 The cable which carries the evacuation signal is damaged. OR

a1.2 Evacuation signal could not be created due to vessel's system. OR

a1.3 The controller is damaged. OR

a1.4 The algorithm of the controller is incorrect.

a2.1 The main inflator is not working. AND

a2.1.1 The cable which carries the signal is damaged. OR

a2.1.2 The inflator itself is damaged. OR

a2.1.3 The controller is damaged. OR

a2.1.4 The algorithm of the controller is incorrect.

a2.2 The pod's inflator is not working.

a2.2.1 The cable which carries the signal is damaged. OR

a2.2.2 The pod's inflator is damaged. OR

a 2.2.3 The controller is damaged. OR

This applies for a3, a4, and a5

a3/4/5.1 Pressure sensor on the pod not working. OR

These apply for a3 and a4

a3/4.2 The cable which carries the signal from the pressure sensor to the encoder is damaged. OR

a3/4.3 The encoder is damaged. OR

a3/4.4 The cables which carry the signals from the encoder to the main controller is damaged. OR

a3/4.5 The cables which carry the signals from the encoder to the main controller is wired incorrectly. OR

a3/4.6 The configuration of the microcontroller is set incorrectly. (e.g. reading D0 instead of D1)

a6.1 Pod separation button not working due to malfunctioning electromechanical (or hydraulic depending on the design) components. OR

a6.1 Pod separation mechanism not working.

a7.1 Wrong wiring of the cables connected to the microcontroller which come from the encoder. OR

a7.2 Pressure sensor may be malfunctioning and sending wrong signals.

These causes are derived by a simple root cause analysis, which should be considered as an example only.

\section{c. Mitigations} areas. a1.1 Mechanically protect cables and put them in safe 
a1.2 The alarm system is out of scope here.

a1.3 Make the main controller redundant and physically separate controllers.

a1.4 Use diverse software in controllers

a2.1 Make the main inflator redundant.

a2.2 The main inflators are more powerful than the pod inflators. The main inflator alone can inflate pods, but it will increase the time of inflation.

a3.2 The pressure is also displayed inside the vessel near the door and a mechanical alternative is built on the door so that the crew can open the door manually.

General: 8 pods are provided in order to accommodate damage or malfunctioning of up to 4 pods. Half of the pods can fail and the personnel are still able to board into the remaining pods.

These are some mitigations possible, which are found in the design. More should be done to get a fully safe system.

\section{CONCLUSIONS}

In the existing systems, the crew is forced to board onto the life-rafts or boats through a slide or a chute. In such case, the personnel become vulnerable to fierce weather conditions by being exposed. Subsequently, these people have to cram into life-rafts/boats. In addition to the drawbacks of their personal discomfort and stress caused by the shock of the events they had been through, they would now have to stay uncomfortably squeezed with other people until a rescue arrives. These adverse outcomes are also mentioned in [16].

In contrast, POMES is designed to grant ease to the crew during and after the evacuation. Most importantly, it is designed to protect the personnel by isolating them inside pods fully. Hence they are not exposed to any external dangers that may come due to bad weather or from marine creatures. Moreover, it is designed taking into consideration personal space which consequently supports the personnel's psychological well-being. Withal, provision of sustenance helps sustaining the crew psychologically and physically. Moreover, there is no possibility of injury during boarding in POMES unlike vertical chute MES [16]. Even injured personnel, if there be any, can board these pods without any difficulties. Moreover, members of the crew with phobias would not be overwhelmed by the fear of chutes or slides.

\section{ACKNOWLEDGEMENTS}

We thank Dr Siewe, without whom ET would not be aware of this conference and we would not have prepared this paper. We thank him for encouraging and supporting the initial idea. We also thank Dr Khan for his aid and availability.

Ultimately, we give special thanks to Mr Michael Physick for his corrections of an early draft of this work and also for his availability.

\section{REFERENCES}

1. Survitec (n.d) Marine Evacuation Systems. [Online] Available at: https://survitecgroup.com/media/342295/survitec mes brochure.pdf [Accessed 15/04/2019]

2. Mercator Media Ltd. (2011) Marine Evacuation Systems: A Viable Alternative To Lifeboats. [Online] Available at: https:// www.motorship.com/news 101/ships-equipment/marine-evacuationsystems-a-viable-alternative-to-lifeboats [Accessed 15/04/2019]

3. Wärstilä (n.d.) Marine evacuation systems (MES). Wärtsilä Encyclopedia of Marine Technology. [Online] Available at: https:// www.wartsila.com/encyclopedia/term/marine-evacuation-systems-(mes) [Accessed 16/04/2019]
4. Lifecraft Systems Australia (n.d.) Marine Evacuation Systems(MES). [Online] Available at: http://www.lsames.com/products/marine-evacuationsystems-mes [Accessed 16/04/2019]

5. Viking Life-Saving Equipment (n.d.) Marine Evacuation Systems. [Online] Available at: https:/www.viking-life.com/en/evacuation-systems-/ marine-evacuation-systems [Accessed 05/05/2019]

6. Det Norske Veritas (2010) Recommended Practice Det Norske Veritas DNV-RP-E403-Hyperbaric Evacuation Systems. [Online] Available at: https://rules.dnvgl.com/docs/pdf/DNV/codes/docs/2010-10/RP-E403.pdf [Accessed 16/04/2019]

7. United States Coast Guard (2017) Marine Evacuation Systems(MES). [Online] Available at: https://www.dco.uscg.mil/Portals/9/ DCO\%20Documents/5p/CSNCOE/FPVE\%20Knowledge/

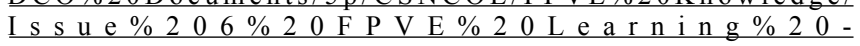
$\% 20 \mathrm{MES} \% 20$ (Marine \%20Evacuation \%20 System).pdf? ver=2017-08-08-074810-337 [Accessed 16/04/2019]

8. Marine Accident Investigation Branch (2003) Report on the investigation of a fatal accident during a vertical chute evacuation drill from the UK registered ro-ro ferry. Report No 18/2003. [Online] Available at: https://assets.publishing.service.gov.uk/media/ 547c70e3e5274a428d0000cb/aquitaine-chute.pdf [Accessed 16/04/2019]

9. RFD (n.d.) Marin Ark Marine Evacuation Systems. [Online] Available at: http://www.shipserv.com/ShipServ/pages/attachments/ 201044/10074\%20Survitec\%20RFD\%20brochure \%20v17.pdf [Accessed $16 / 04 / 2019]$

10. University of Wisconsin (n.d.) Aramid Fibres. [Online] Available at: https://www.chem.uwec.edu/chem405_s01/malenirf/project.html [Accessed 18/04/2019]

11. DuPont (2019) Better, Stronger and Safer with Kevlar ${ }^{\circledR}$ Fiber. [Online] Available at: http://www.dupont.com/products-and-services/ fabrics-fibers-nonwovens/fibers/brands/kevlar.html [Accessed 18/04/2019]

12. IEC (2010) International Electrotechnical Commission 61508 Functional safety of electrical/electronic/programmable electronic safetyrelated systems-Parts 1-7. Geneva: International Electrotechnical Commission, ed. 2, 2010.

13. ISO (2017) ISO/IEC/IEEE 12207:2017 Systems and software engineering -- Software life cycle processes. Geneva: International Organization for Standardization, 2017.

14. Breinholt, C.; Ehrke, K.; Papanikolaou, A.; Sames, P.; Skjong, R.; Strang, T.; Vassalos, D.; Witolla, T. (2012) SAFEDOR - The Implementation of Risk-Based Ship Design and Approval. Procedia Social and Behavioral Sciences 48 (2012), pp. 753-764.

15. Harwood, D.; Farrow, Al. (2008) Factors Associated With Marine Injuries During Vertical Chute Evacuation Training. Occupational Medicine 58 (2008), pp. 400-405.

16. Harwood, D.; Farrow, A. (2012) Validation of Reported Events From A Vertical Chute Marine Evacuation Study. Applied Ergonomics 43 (2012), pp. 1105-1109.

17. Hearle, J. (2001) Textile Fibers: A Comparative Overview. Encyclopedia of Materials: Science and Technology. 2nd ed. UMIST, Manchester, UK.

18. Yang, S.; Chalivendra, V.; Kim, Y. (2017) Fracture and Impact Characterization of Novel Auxetic Kevlar/Epoxy Laminated Composites. Composite Structures 168 (2017) pp. 120-129.

19. DuPont De Nemours \&Co (2008) DUPONT: Garments made of NOMEX Comfort provide enhanced protection for multi-task utility workers at German City Council. M2 Presswire. Coventry, UK: Normans Media Ltd. 